\title{
HIGH CURRENT PULSE STRIPLINES
}

\author{
K. Bourkland, C. Jensen, D. Tinsley, Fermilab*, Batavia IL USA 60510 \\ W. Markel, Markel \& Associates, Orland Park, IL 60462
}

\section{Abstract}

There are two different horn systems under construction at Fermilab for neutrino beamlines. The NuMI project requires a power supply that operates at $970 \mathrm{~V}, 205 \mathrm{kA}$, $2.6 \mathrm{~ms}$, and $0.53 \mathrm{pps}$. The MiniBooNE project requires a power supply that operates at $5.5 \mathrm{kV}, 170 \mathrm{kA}, 140 \mu \mathrm{s}$ and 5 pps. Both require long low inductance connections between the power supply and horn; 60 feet for MiniBooNE and 230 feet for NuMI. This paper discusses several electrical and mechanical design requirements that have been overcome. These include low impedance, radiation hardness, voltage holdoff, clamping for electrical and mechanical connections and humidity and dust control. Measurements of the inductance of the striplines and voltage holdoff will be compared to calculations. The results of some tests will be discussed.

\section{INTRODUCTION}

Fermilab has two new neutrino beamlines which require focusing of the particle shower after the target. NuMI uses $120 \mathrm{Gev}$ protons on target and has detectors both on site and $600 \mathrm{~km}$ away in Minnesota. MiniBooNE uses $8 \mathrm{Gev}$ protons on target and has a detector on site only. The striplines near their respective target vaults have high radiation dose rates (estimated at $10^{\wedge} 4 \mathrm{rad} / \mathrm{hr}$ for MiniBooNE, $10^{\wedge} 6 \mathrm{rad} / \mathrm{hr}$ for NuMI) and this poses some additional constraints in addition to the high peak and rms currents. Also, since it is anticipated that the horn may fail in the lifetime of the experiment, the striplines must be remotely disconnected from the horn before the horn can be stored in a sarcophagus. Finally, the environmental conditions in the target area can include water condensation and rock dust build up.

There are several properties the stripline connecting the power supply to the horn must have. It must have a known and relatively low inductance, it must not have voltage breakdown, it must last for the life of the experiment without failure from either mechanical or radiation fatigue and it must have relatively low ohmic losses. Substantial work was done to simulate and measure several characteristics of the striplines. The primary electrical differences between MiniBooNE and NuMI are pulse length, repetition rate and operating voltage. This has lead to different design choices in several areas. Each stripline has to be designed as an electromechanical system. Each system had to find a way to overcome 1) high radiation dose, 2) high mechanical forces and vibration, 3) moderate power dissipation in the stripline, 4) moderate

* Fermilab is operated by Universities Research Association Inc. under Contract No. DE-AC02-76CH03000., electrical insulation 5) joints that are field assembled and 6) moisture and dust in the environment. The way these two striplines accomplished there goals are shown in cross section, Figures 1 and 2. They are to the same scale and are oriented as they will be installed.

There are several concerns common to both projects. This has led to some commonality. The NuMI design was driven by a substantial rms current and so required very good conductor. Both projects requested a low mass transmission line near the targets to reduce the residual radiation rate. This should make it easier to remove a failed horn. For these and economic reasons, both projects use high purity 6101-T61 Aluminum alloy for the conductor, 3/8" thick x 12 " wide. The radiation rates also precluded any carbon based insulation. The mechanical forces involved required a substantial clamping force. For these reasons, both projects use a type of ceramic for insulation.

Classic equations for high frequency stripline applications typically neglect skin depths since they are negligible compared to conductor spacing. The classic equation for the calculation of stripline inductance;

$$
L=\mu_{0} \frac{a}{b}
$$

where $a=$ the air gap and $b=$ the conductor width, incorrectly predicts the inductance for the NuMI sample to be $82 \mathrm{nH} /$ meter. It also fails to predict the correct inductance value for the MiniBooNE stripline.

\section{MINIBOONE DESIGN}

The high voltage holdoff, $7 \mathrm{kV}$, and long lifetime, $2 \times 10^{\wedge} 8$ pulses, required for MiniBooNE were the driving requirements. However, the first decision that was made was the number of conductors. There was considerable discussion about using an odd number of conductors. The outer conductors carry only half the current and this leads to a much reduced force on them. The magnetic fields external to the stripline are also much reduced leading to a much smaller inductance. For the conductor spacing and width used in this instance, the use of a balanced transmission line reduced the inductance to $55 \%$ of the unbalanced case and reduced the force on the outside conductors to $30 \%$ of the unbalanced case. In addition, there is no net force on the internal conductors.

Having decided the topology, the design for high voltage holdoff was next. Initially, several materials were investigated for the insulating spacers; polyimide $\left(\varepsilon_{\mathrm{r}}=4.2\right)$, boron nitride $\left(\varepsilon_{\mathrm{r}}=3.7\right)$, alumina $\left(\mathrm{Al}_{2} \mathrm{O}_{3}\right) \quad\left(\varepsilon_{\mathrm{r}}=9.9\right)$ and Zirconia $\left(\mathrm{ZrO}_{2}\right) \quad\left(\varepsilon_{\mathrm{r}} \sim 26\right)$. After several electric field 
simulations with the different materials it was clear that a different shape would be required in the each case.

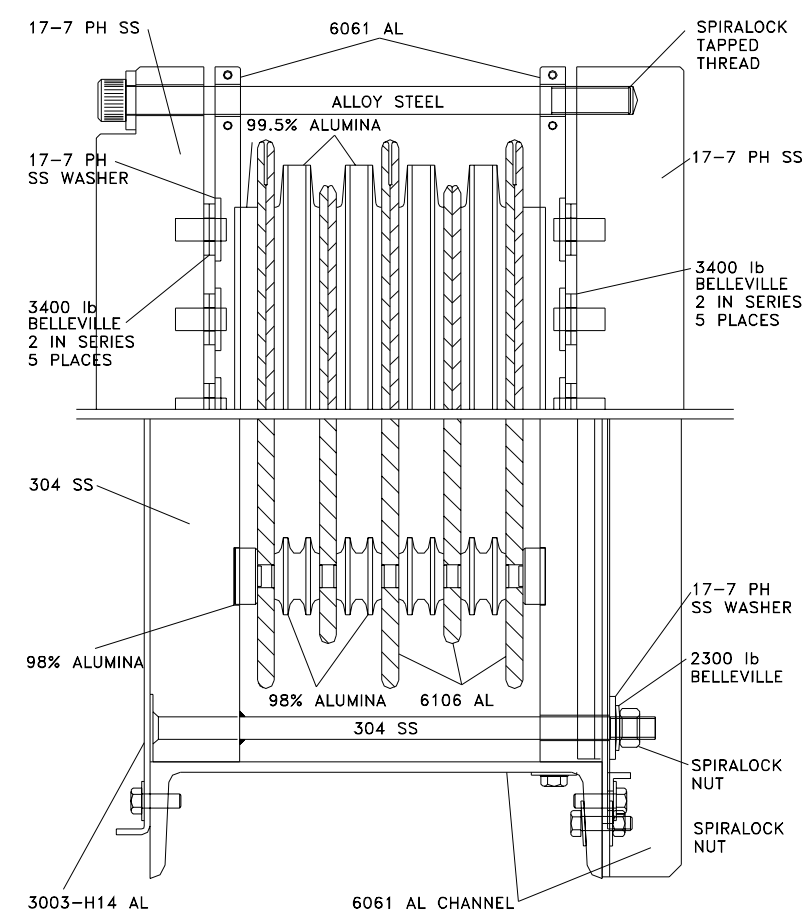

Figure 1. Cross-sectional view of MiniBooNE stripline Upper portion of figure shows cross section at a typical joint held by spring style clamp. Lower portion of figure illustrates the mid-line style of clamp and surrounding cooling air plenum. $\mathrm{L}=18.5 \mathrm{nH} /$ meter. $\mathrm{R}_{\mathrm{AC}} \approx 15 \mu \Omega /$ meter.

As a further constraint boron nitride and zirconia are more expensive than alumina $\left(98 \% \mathrm{Al}_{2} \mathrm{O}_{3}\right)$. Also, the use of polyamide would have been restricted to low radiation areas. Because different materials would have required different designs, and since the initial quote for alumina insulators was modest, $\$ 10$ each, the decision was made to use alumina everywhere.

The nominal operating current for the horn is $170 \mathrm{kA}$ which corresponds to approximately $5.5 \mathrm{kV}$. The design is to allow up to $250 \mathrm{kA}$, which would raise the voltage to $8 \mathrm{kV}$ and $7.5 \mathrm{pps}$. Additionally, there is a high charged particle flux $\left(\sim 10^{\wedge} 13\right.$ protons over $\left.1.2 \mathrm{us}\right)$ passing next to a portion of the stripline which could induce breakdown and arc formation. Therefore, the stripline was designed with a 1 inch spacing and a average electric field of $10 \mathrm{~V} / \mathrm{mil}(400 \mathrm{~V} / \mathrm{mm})$.

Given the material and average electric field of $10 \mathrm{~V} / \mathrm{mil}$, a detailed simulation of the strip line was done using Opera-2D. It was found that even with the balanced stripline topology, there were significant electric and magnetic fields within several $\mathrm{cm}$ of the return conductors. Additionally, even without the insulating spacers, the maximum field stress on the edge of the supply conductors was over $20 \mathrm{~V} / \mathrm{mil}(790 \mathrm{~V} / \mathrm{mm})$. Insetting the supply conductor by $50 \%, 100 \%$ and $150 \%$ of the conductor spacing was simulated. With no inset, the electric and magnetic fields at the outside edge of the return conductor were $~ 90 \%$ of the average field between conductors. With an inset if $1 / 2$ " they were $\sim 25 \%$ of average. With an inset of 1 " they were $\sim 10 \%$ of average and this was chosen for the design. The maximum field on the supply conductor was not reduced, but the influence of external components (clamps for restraining and ductwork for cooling) was greatly reduced.

Several alumina insulator designs were examined next. The electrical design criteria was always the same, to keep the peak voltage stress to no more than $\sim 20 \mathrm{~V} / \mathrm{mil}$ in the air (which is present all along the edge of the supply conductor) and a minimum creep path of $5 \mathrm{~V} / \mathrm{mil}$ (200 $\mathrm{V} / \mathrm{mm}$ ) with $10 \mathrm{kV}$ between conductors. The mechanical design had to be manufacturable and avoid creating large shear forces which would fracture the alumina. The breakdown voltage in air with a uniform field at STP is approximately $72 \mathrm{~V} / \mathrm{mil}$ and the creapage path for outdoor insulators is typically twice the air path length. Our design criteria are indeed conservative, however it will be impossible to repair or replace the stripline after it is installed. The final design is shown in Figure 1. Two such assemblies are used in parallel to power the horn.

There are two styles of clamps for this stripline, a rigid bar style for mid-line locations, and a spring loaded style at the electrical joints. A simulation of magnetic fields was used to determine that the peak force on each of the outside return conductors is approximately $680 \mathrm{~N} / \mathrm{m}$ of length, or approximately 2200 Pascal ( $0.32 \mathrm{psi}$ ). The clamping force on the first type is $4600 \mathrm{lbs}$ and the clamp spacing is 18 ". The clamping force on the joint clamp is $17,000 \mathrm{lbs}$ and surface area directly under this force is $11.3 \mathrm{in}^{2}$, giving a pressure on the conducting surface of 1500 psi. The rms current density in this surface is $70 \mathrm{Arms} / \mathrm{in}^{2}\left(11 \mathrm{Arms} / \mathrm{cm}^{2}\right)$ and the peak current density is $3,800 \mathrm{Apk} / \mathrm{in}^{2}\left(580 \mathrm{Apk} / \mathrm{cm}^{2}\right)$.

\section{Testing}

Finally, a test stripline was manufactured and the corona inception and breakdown voltages were measured. The tests were done in July and August in an airconditioned room with an air temperature of $75^{\circ} \mathrm{F}$. Initially, the test stripline had several sharp edges on the aluminium bus that were then removed. The test stripline was approximately 48 inches long and contained three sets of insulator and clamp. Corona discharges of $100 \mathrm{pC}$ typically started at $\sim 15 \mathrm{kV}$ rms, $60 \mathrm{~Hz}$. This corresponded to an average field between the conductors of $20 \mathrm{~V} / \mathrm{mil}$ $(800 \mathrm{~V} / \mathrm{mm})$.

An arcing breakdown occurred at $21-22 \mathrm{kV}$ rms. The arcing breakdown did not occur at the mid-line clamp insulators. The supply conductor in the test stripline has two edges that meet to form a corner and the breakdown occurred there. Additional filing and polishing did not raise the breakdown voltage substantially. 
Another test was done with a simple straight cylinder alumina insulator. The corona discharge then started at $10 \mathrm{kVrms}$ and breakdown was along the straight insulator at $21 \mathrm{kVrms}$.

The final high voltage testing was done with a joint clamp. The corona inception was at $\sim 14 \mathrm{kVrms}$ and breakdown was again near $22 \mathrm{kV}$ rms. This time there was breakdown alternatively between the corner where it had happened previously and across the alumina bar insulator where the clamped joint was made.

The final stripline was then manufactured. The longest length was approximately 8 meters. The inductance of this length at $3.5 \mathrm{kHz}$ was measured with an HP4194 impedance bridge to be $18.5 \mathrm{nH} / \mathrm{m}$. This is $28 \%$ larger than the Opera-2D magnetic field simulation of $14.5 \mathrm{nH} / \mathrm{m}$. The classis equation for a stripline predicts an inductance of at least $30 \mathrm{nH} /$ meter. This was a difficult measurement to make and there might be considerable error from the connections to all the buses.

The DC resistance of a silver plated clamped joint was also measured as a function of force with a 100 Amp Biddle Ohmmeter. The joint resistance was on the order of $1 \mu \Omega$ and changed less than $20 \%$ between a clamp pressure of $700 \mathrm{psi}$ and 1,500 psi.

The finished stripline has six sections. A typical section is 10 feet long, has 7 mid-line clamps, 1 joint clamp, 1 bend, and costs $\sim \$ 13,000$. The bus material, machining, welding, and plating is $40 \%$ of the cost, the joint clamp is $20 \%$, all of the mid-line clamps are $15 \%$, and the remainder is for the air duct.

\section{NUMI DESIGN}

From Figure 2, it can be seen that the NuMI stripline is an assembly of eight layers of conductor, 3/8" thick by 12 " wide, with 3/8" airgaps. Each conductor pair is driven by one-quarter of the capacitor bank.

Because the maximum operating voltage in this application is $1 \mathrm{kV}$ or less and the rms current higher than for MiniBooNE, it was elected to make all conductors of equal width to achieve the lowest resistance. This design is to accommodate pulse widths of up to $5.2 \mathrm{~ms}$ in the future, increasing the rms current.

The fundamental frequency of the NuMI half-sine pulse is $100 \mathrm{~Hz}$, necessitating an understanding of the methods for calculating inductance of striplines at low frequencies.

Measurements were pursued using an 18 foot long, 6" wide, sample stripline of the same aluminum alloy, conductor thickness, and conductor spacing. Three methods were used to measure the inductance; From V and I values during resonant discharge, $\mathrm{V}$ and I values during $\mathrm{AC}$ excitation at $100 \mathrm{~Hz}$, and $\Delta \mathrm{T}$ of resonant period. The following measured values per meter are for the conductor pair.

The first method involved installing the sample stripline into a resonant R-L-C circuit that could pulse it with a $5.2 \mathrm{~ms}$, half-sine pulse, and as high as $25 \mathrm{kA}$ peak for additional thermal studies. The stripline was connected in series with a $40 \mu \mathrm{H}$ inductor to achieve the desired

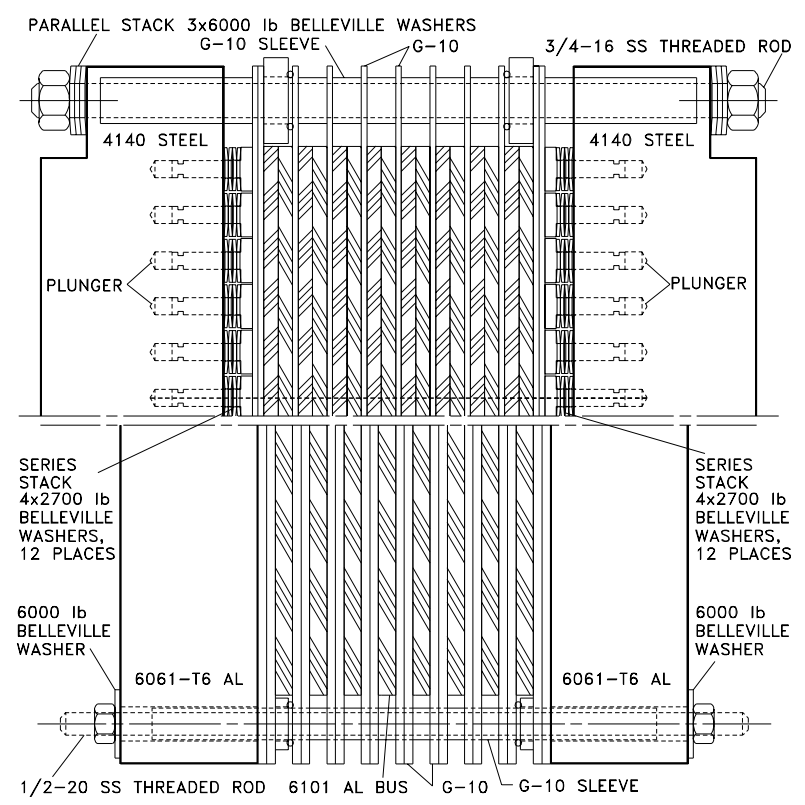

Figure 2. Cross-sectional view of the NuMI stripline. Upper portion of figure shows the stripline cross section at a typical joint, held at $28,000 \mathrm{lbs}$ by the spring style bar clamp. Lower portion of figure illustrates the mid-line style of aluminum bar clamp. $\mathrm{L}=16 \mathrm{nH} / \mathrm{m}, \mathrm{R}_{\mathrm{AC}}=10 \mu \Omega / \mathrm{m}$.

resonant frequency. A $20 \mathrm{mF}$ capacitor bank and SCR switch complete the circuit. Voltage and current were monitored, respectively, across and through the stripline sample. Measurements were taken at maximum di/dt and at $\mathrm{di} / \mathrm{dt}=0$. Fitting to the equation;

$$
e=L \frac{d i}{d t}+I R
$$

$\mathrm{L}$ was found to be $87 \mathrm{nH} /$ meter and $\mathrm{R}_{\mathrm{AC}}, 60.4 \mathrm{u} \Omega /$ meter. Note, however, that at time $=0, \mathrm{di} / \mathrm{dt}$ maximum, the current is zero and no penetration of current into the conductor has taken place. Therefore, the value for $\mathrm{L}$ calculated by this method is the effective inductance at time $=0$ only. The $R_{A C}$ value, however, will be valid. The measured $R_{D C}$ was $45 \mu \Omega /$ meter.

The second method involved driving the stripline sample with a power oscillator, followed with a matching transformer and a $500 \mu \mathrm{H}$ series inductor, to achieve currents of order $100 \mathrm{~A}$ at $100 \mathrm{~Hz}$. From the measured voltage across, and current in the stripline, the impedance was calculated. Using this impedance value along with the $R_{A C}$ value from the first measurement method, $X_{L}$ was calculated. The inductance was determined to be $130 \mathrm{nH} /$ meter.

The final method again involved operating the sample in the pulser circuit. A measurement was made of the difference in the half-sine period of the pulse with, and without, the stripline sample included in the circuit. Since 
the predominant resistance in the circuit is in the $40 \mu \mathrm{H}$ inductor, the resistance of the stripline was neglected in the following calculation. From the measured change in period and the equations;

$$
\begin{aligned}
& T=\pi \sqrt{L C} \\
& \Delta T \approx \frac{\pi}{2} \sqrt{\frac{C}{L_{\text {coil }}}} \bullet L_{\text {stripline }}
\end{aligned}
$$

for the half-sine case, the value of $\mathrm{L}$ for the stripline is obtained. Eq. 4 yields an inductance value of $126 \mathrm{nH} /$ meter.

From these measurements it was learned that Eq. 1 fails to predict our measured values. With a skin depth of 0.315 " in the bus material, an air gap of 0.375 " and a conductor thickness of 0.375 ", skin depth was a significant factor. For calculating inductance we use the same assumption as equation 1, except we take into account that the b-field is constant and maximum in the air gap, and decreasing to zero within the conductors by 1 skin depth. By integrating all of the field in the conductors and the air gap it can be shown that;

$$
L=\mu_{0} \frac{a+2 / 3 \delta}{b},
$$

where $a$ is the air gap, $b$ is the conductor width, and $\delta$ is the skin depth. This equation predicts the sample stripline inductance to be $126 \mathrm{nH} /$ meter, in agreement with the measurements.

The Opera-2D simulation predicted inductance to be $121 \mathrm{nH} /$ meter and resistance as $81 \mu \Omega /$ meter. The simulation was done assuming a steady state AC solution of $120 \mathrm{~Hz}$. The energy stored and the energy lost were computed and then the inductance and resistance were calculated from those numbers. The program estimated its own error from this simulation at 3\%. Simulation of the entire eight conductor stripline using the same method gave an inductance of $15 \mathrm{nH} / \mathrm{m}$ and $10 \mu \Omega / \mathrm{m}$ with a program estimated error, again, of $3 \%$.

Higher rms current requirements for NuMI, 7,250 A vs: 3,200 A for MiniBooNE, lead to effort in joint design. The extruded bus material was purchased in 35 foot lengths, as long as possible to minimize the number of joints and short enough to facilitate handling. With a need to construct 230 feet of stripline, a method was developed to clamp pre-fabricated sections together. The joint compression clamp illustrated in Figure 2 consists of two 4140 alloy steel bars, 1.5 " thick x 4" high x 15" long, pulled together by two 3/4" diameter threaded rods. Twelve independent plungers, backed by conical spring washers, in each bar assure the development of uniform pressure distribution across the length of the clamp and against the stripline conductors. The clamps aretightened to $28,000 \mathrm{lbs}$. and are capable of $32,000 \mathrm{lbs}$ maximum force. This translates to $3,500 \mathrm{psi}$ in the contact region of the stripline conductor, each layer carrying 1,810 Arms, and a current density in the joint of 193 Arms/in².
The mating surfaces of the joint region of bus conductor are machined with a shallow face cut in to assure flatness. A bevel is also machined on the bus ends to facilitate joint engagement. The joint regions are then Silver plated to a thickness of 0.0002" to assure low ohmic contact and protection from oxidation. Joints of this type have successfully operated for $2 \times 10^{\wedge} 6$ shots to date in our horn testing program.

Bolted joints with clamping forces of 4,000 psi, using the same joint region preparation methods, have also been successfully tested to $1.5 \times 10^{\wedge} 6$ shots in our SCR test fixture at the same current densities. DC resistance measurements show the joint to be of the order of $1 \mu \Omega$ on the 6" wide sample and stable throughout the testing. Current densities were $6.2 \mathrm{kApk}$ and $225 \mathrm{Arms} / \mathrm{in}^{2}$

In the regions between joints, bar clamps consisting of two pieces of aluminum bar stock, 1.5 " x 3" cross-section and 15 " long, held with two $1 / 2$ " threaded rods, provide a clamping force 6,000 lbs. Initial spacing of these mid-line clamps has been empirically established at 12 ". Vibration studies are underway and may suggest changes to this interval. Insulation between conductors in the non radiation areas will be of G-10 and Kapton ${ }^{\circledR}$. In the high radiation areas of the beamline, Alumina will be the material of choice.

The electrical connection of the stripline conductors to the face of the horn is via bolted joints. The stripline fans out into conductor pairs to supply current to the horn at four points about its circumference. Sixteen raised bosses on the end face of the horn provide contact regions for each respective stripline, two bosses per conductor. The electrical contact area at each boss is $1.15 \mathrm{in}^{2}$ and all contact surfaces, horn and stripline, are silver plated to 0.0002 " thickness. The contact pressure at each boss is 8,000 psi. For the present, the horn testing program is being carried out with the temporary use of a pulser limited to $850 \mu$ s half-sine pulses, but able to operate at $205 \mathrm{kApk}$. This yields a current density at each contact of $22 \mathrm{kApk} / \mathrm{in}^{2}$. However, our experience to date with the horn-stripline joints is limited by this supply to just 330 Arms/in ${ }^{2}$. Ultimately 790 Arms/in ${ }^{2}$ will be achieved with a new power supply presently under construction. Since the horn is water cooled, no significant contact heating has been experienced. Testing to $2 \times 10^{\wedge} 6$ shots, to date, has been successful at this limited level.

\section{ACKNOWLEDGEMENTS}

The authors wish to acknowledge the participation of Dan Wolff for his suggestion of treating the stripline as a single turn magnet for the purpose of calculating inductance; Howie Pfeffer for his input and many valuable suggestions during the course of this work; and the technical support of John Ellis. 